\title{
RELACIONES TERRITORIALES DE LA ACTIVIDAD TURÍSTICA EN TORNO AL PARQUE NACIONAL RINCÓN DE LA VIEJA, COSTA RICA
}

\section{TERRITORIAL RELATIONS OF THE TOURIST ACTIVITY AROUND RINCÓN DE LA VIEJA NATIONAL PARK, COSTA RICA}

\author{
Pablo Miranda-Álvarez ${ }^{1}$ \\ Meylin Alvarado-Sánchez ${ }^{2}$ \\ Universidad Nacional de Costa Rica, Costa Rica
}

\section{RESUMEN}

El desarrollo de la actividad turística en Costa Rica ha estado ligado al Sistema Nacional de Áreas de Conservación del país, el cual ha servido como base para el desarrollo de su imagen turística a nivel internacional. Como resultado de este desarrollo, la Unidad Turística Pacífico Norte establecida por el Instituto Costarricense de Turismo ha experimentado el mayor crecimiento del país desde la década de 1990 al ofrecer productos de sol y playa junto con áreas protegidas generando un aumento en la visitación al Parque Nacional Rincón de la Vieja y el establecimiento, en las comunidades aledañas al área protegida, de una serie de empresas de hospedaje que ofrecen diversas actividades relacionadas con el segmento de turismo alternativo. Por tal razón, se presenta una caracterización del sistema turístico asociado con el Parque Nacional Rincón de la Vieja que permite

1 Geógrafo, Licenciado en Ordenamiento Territorial, Máster en Gestión de Turismo de Naturaleza, académico de la Escuela de Ciencias Geográficas de la Universidad Nacional de Costa Rica (UNA), Costa Rica. Correo electrónico: pmiranda@una.cr

2 Geógrafa, Licenciada en Educación Ambiental, Máster en Desarrollo Rural Comunitario, académica de la Escuela de Ciencias Geográficas de la Universidad Nacional de Costa Rica (UNA), Costa Rica. Correo electrónico: malvara@una.cr

Fecha de recepción: 25 de agosto de 2016

Fecha de aceptación: 30 de noviembre de 2016 
Pablo Miranda-Álvarez, Meylin Alvarado-Sánchez. Territorial relations of the tourist activity around Rincón de la Vieja National Park, Costa Rica

identificar las características de la oferta, demanda, el espacio geográfico y los agentes del mercado con el fin de establecer las relaciones territoriales con las comunidades aledañas al área protegida.

Palabras clave: turismo, Geografía del turismo, área protegida, sistema turístico.

\begin{abstract}
The development of the tourist activity in Costa Rica has been related to the Conservancy Areas National System (SINAC), which has been the basis for developing its tourism image at an international level. As a result of this development, the Northern Pacific Tourism Unit -established by the Costa Rican Tourism Institute, has experienced the greatest growth of the country since the 1990's by offering sun and beach products along with protected areas, thus increasing the visits to the Rincón de la Vieja National Park and establishing, in the communities around the protected area, as series of lodging companies related to the alternative tourism segment. Therefore, a characterization of the tourism system related to the Rincón de la Vieja National Park is presented, and it allows identifying the characteristics of the supply, demand, geographical space, and the market agents in order to establish territorial relations with the communities near the protected area.
\end{abstract}

Keywords: tourism, tourism geography, protected area, tourism system.

\title{
Introducción
}

Desde mediados de la década de 1980, el turismo se ha convertido en uno de los sectores más dinámicos de la economía costarricense, superando las divisas generadas por los productos de exportación tradicionales como el banano y el café (ICT, 2015). En este periodo, el país comienza a consolidarse como uno de los primeros destinos ecoturísticos a nivel internacional, situación que posiciona privilegiadamente a Costa Rica como destino turístico.

A partir de los años noventa, la actividad turística tiene un papel preponderante en la economía del país, pasando de recibir 500000 visitantes en el año 1991 a 2526817 en el año 2014 lo cual generó ingresos por \$ 2526 millones, equivalente al 5,3\% del Producto Interno Bruto del país (ICT, 2015).

Con el fin de planificar el desarrollo de la actividad, el Instituto Costarricense de Turismo (ICT), en su Plan General de Desarrollo Turístico Sostenible 2002-2012, define y delimita diez unidades de planeamiento territorial (ICT, 2002). Entre ellas, sobresale la Unidad de Planeamiento Guanacaste Norte (UPGN), localizada en la provincia de Guanacaste en la Vertiente del Pacífico costarricense, donde se ubica la ciudad de Liberia como centro urbano principal- 
A su vez, el Sistema Nacional de Áreas de Conservación (SINAC) "es el principal bioactivo que soporta la estrategia de desarrollo ecoturístico en el país" (Arias et al, 2014,276) y concentra las áreas silvestres protegidas más visitadas entre ellas el Parque Nacional Rincón de la Vieja (PNRV), fundado en el año 1974, siendo uno de los atractivos turísticos más importantes de la Unidad Turística Guanacaste Norte al complementar el segmento de sol y playa que es el producto turístico más destacado. Por su parte, las comunidades aledañas al parque nacional se han convertido en punto de atracción para el establecimiento de empresas de hospedaje, especialmente por los recursos naturales y culturales que permiten el desarrollo del turismo alternativo.

\section{Objetivo general}

Revelar las relaciones territoriales de la actividad turística en torno al Parque Nacional Rincón de la Vieja, Costa Rica.

\section{Caracterización físico - geográfica del área de estudio}

Fundado en noviembre de 1862 (IFAM, 1989) el cantón de Liberia (donde se localiza el área de estudio), posee un área de $1567 \mathrm{~km}^{2} \mathrm{se}$ conforma de cinco distritos (Liberia, Cañas Dulces, Mayorga, Nacascolo y Curubandé), como lo muestra el siguiente mapa. Cuenta con una población para el año 2011 de 62987 habitantes (INEC, 2012) y un índice de desarrollo humano de 0,817 , ubicándose en el puesto 24 de los 81 cantones del país (PNUD, 2011). 
Pablo Miranda-Álvarez, Meylin Alvarado-Sánchez. Territorial relations of the tourist activity around Rincón de la Vieja National Park, Costa Rica

Mapa 1. Ubicación del área de estudio

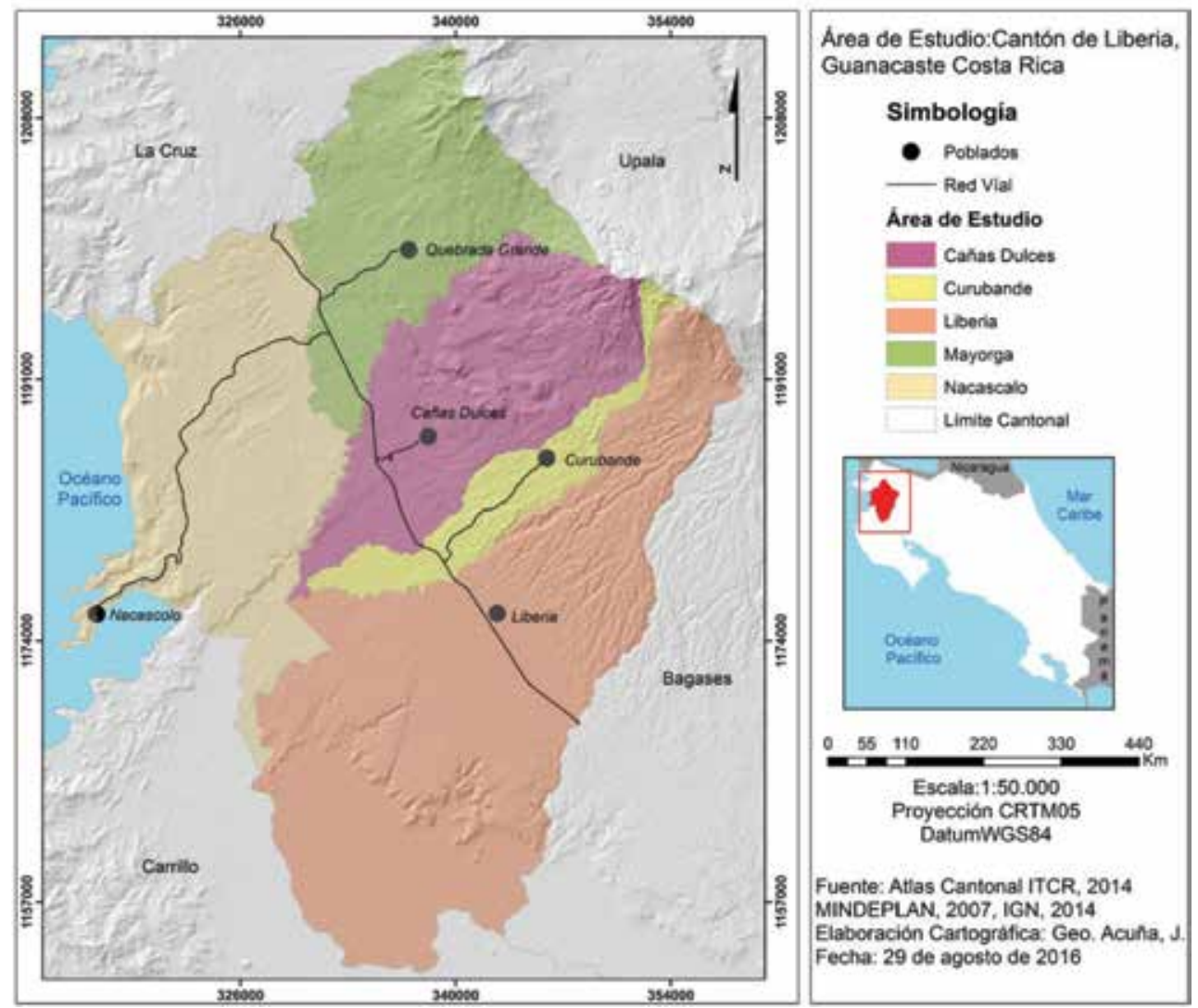

Geológicamente se encuentra constituida por materiales de los períodos Cretácico Terciario y Cuaternario; se destacan las rocas de las unidades Complejo de Nicoya, Formación Montezuma, Formación Brito y Edificios Volcánicos recientes y actuales (Denyer, 2000). Geomorfológicamente, en el cantón se encuentran tres subunidades, la primera donde se encuentra el área de estudio denominada Formas de origen volcánico, que se divide en dos subunidades: Meseta de Santa Rosa y Sierra Volcánica de Guanacaste, dentro de esta última se destaca la Sierra Volcánica de Guanacaste, representada por los macizos volcánicos Rincón de la Vieja - Santa María, y Cacao. La segunda, llamada Sedimentación aluvial, correspondiente a las partes bajas del cantón; y la tercera conocida como Denudación en Rocas Igneas, dividida en dos subunidades: Laderas muy 
Empinadas y Escarpes de Erosión del Frente de la Meseta de Santa Rosa, y Relictos Volcánicos de los Cerros Góngora, Cañas Dulces y San Roque (IGN, 1978).

La temperatura anual varía entre $\operatorname{los} 18^{\circ} \mathrm{C}$ en las partes altas y los $28^{\circ}$ $\mathrm{C}$ en las zonas bajas del cantón, siendo los meses más cálidos marzo, abril y mayo, y los más frescos noviembre, diciembre y enero; la precipitación media anual se encuentra entre los 2000 y $6000 \mathrm{~mm}$ según la zona (IFAM, 1989). Según la clasificación de zonas de vida para Costa Rica elaborada por Holdridge, se identifican: bosque pluvial sempervirente montano bajo, bosque húmedo sempervirente submontano, bosque semideciduo estacional submontano. La fauna abarca gran cantidad de aves, mamíferos, reptiles e insectos, especialmente en el Parque Nacional Rincón de la Vieja, Parque Nacional Santa Rosa, Parque Nacional Guanacaste (Vargas, 1997).

\section{Referente conceptual}

El turismo, definido por la Organización Mundial del Turismo (OMT) como "las actividades que realizan las personas durante sus viajes y estancias en lugares distintos al de su entorno habitual, por un periodo de tiempo consecutivo inferior a un año con fines de ocio, por negocios y otros motivos" (Sancho, 2001:21), actualmente se encuentra en un proceso constante de consolidación, el cual incluye su carácter multidisciplinar dando como resultado una amplia gama de definiciones relacionadas con las diversas perspectivas de las disciplinas que lo estudian (Monterrubio, 2011). Por su parte Velasco (2013:17) indica que "cada investigador aborda el turismo desde las teorías y conceptos propios de su disciplina y señala tres posibles definiciones de turismo: las definiciones que se realizan observando el turismo desde una disciplina académica concreta, destacando la economía y la geografía entre muchas otras, las que se centran en las características del turista, útiles desde el punto de vista estadístico y Las que parten de la idea de sistema visto como un conjunto de elementos que se relacionan y generan procesos dinámicos, permitiendo centrarse en los elementos específicos como en las dinámicas de relación entre ellos"

Desde la geografía, el estudio del turismo se encuentra relacionado con el territorio, como una actividad que se desarrolla "en un rango o escala geográfica, fundamentalmente regional, nacional o internacional, a diferencia de otras actividades recreativas de carácter doméstico, habitual 
o de salidas diarias, puesto que la actividad turística se caracteriza por un desplazamiento del lugar de residencia habitual y una duración mínima del mismo" (Vera et al, 2013, p. 80). Por estas razones, Lozato-Giordat (1990, p.13) define a la geografía del turismo como "el estudio de las relaciones entre el espacio y las actividades turísticas [...] sin descuidar por ello los demás factores que intervienen en el proceso turístico". La geografía del turismo utiliza tres herramientas (dos de ellas tomadas de la geografía) que permiten abordar el estudio de la actividad turística: las escalas territoriales, el análisis de la diversidad ambiental, social y económica de los territorios dedicados al turismo y la articulación como espacio emisormovilidad-destino turístico (Vera et al, 2013) e indica que, al igual que otras ramas de la geografía, el estudio de las actividades turísticas a través de los años ha pasado de un tratamiento descriptivo a uno explicativo.

El destino turístico, definido como "un espacio geográfico determinado con rasgos propios y servicios, y con cierta capacidad administrativa para desarrollar instrumentos comunes de planificación, que adquiere centralidad atrayendo a turistas mediante productos perfectamente estructurados y adaptados a las satisfacciones buscadas, gracias a la puesta en valor y ordenación de los atractivos disponibles; dotado de una marca, y que se comercializa teniendo en cuenta su carácter integral" (Valls, 2004:1718). Este destino se estructura a partir de tres componentes: el primero de ellos conformado por uno o varios elementos nucleares principales que responden a la experiencia buscada por la demanda; el segundo por una serie de elementos periféricos (infraestructura, alojamiento, alimentación, información) directamente unidos a los anteriores y que estructuran la experiencia y, por último, una serie de elementos complementarios (recursos secundarios) que en conjunto con los anteriores configuran nuevos productos turísticos (Valls, 2014).

Como parte de un destino turístico, las áreas protegidas entendidas según la UICN y citado por Dudley (2008:10) como "un espacio geográfico claramente definido, reconocido, dedicado y gestionado, mediante medios legales u otros tipos de medios eficaces para conseguir la conservación a largo plazo de la naturaleza y de sus servicios eco sistémicos y sus valores culturales asociados" y cuyos objetivos incluyen: conservar la composición, estructura, función y potencial evolutivo de la biodiversidad, contribuir a las estrategias de conservación regionales, mantener la 
diversidad de paisajes, hábitats y ecosistemas asociados, así como, beneficiar a las comunidades residentes o locales

Hoy en día, y particularmente en el caso de Costa Rica, la actividad turística ha crecido y se ha consolidado en torno a las áreas naturales protegidas y su entorno, donde se pueden realizar diversos tipos de turismo que guardan relación entre sí, siendo el caso del turismo alternativo, definido por la SECTUR (2004:22) como "los viajes que tienen como fin realizar actividades recreativas en contacto directo con la naturaleza y las expresiones culturales que le envuelven con una actitud y compromiso de conocer, respetar, disfrutar y participar en la conservación de los recursos naturales y culturales" dentro de esta categoría se incluyen el ecoturismo, el turismo de naturaleza y el turismo rural. El primero de ellos se define como "un segmento del turismo sostenible que ofrece experiencias que permiten a los visitantes descubrir áreas naturales mientras preservan su integridad, así como entender, a través de la interpretación y la educación, el significado natural y cultural del lugar" (Goelder, 2011:543).

Por su parte la SECTUR (2007:3), define el turismo de naturaleza como "aquellos viajes que tienen como fin realizar actividades recreativas en contacto directo con la naturaleza y con las expresiones culturales de un lugar, con una actitud y compromiso de conocer, respetar, disfrutar y participar en la conservación de los recursos naturales y culturales" y el turismo rural comunitario como "Experiencias turísticas planificadas e integradas sosteniblemente al medio rural y desarrolladas por los pobladores locales organizados para beneficio de la comunidad" (Asamblea Legislativa de Costa Rica, 2007).

Aunque el espacio es parte fundamental de la experiencia turística para su análisis desde una perspectiva territorial, la teoría de sistemas complejos juega un papel primordial ya que "por las características propias del objeto de estudio de la geografía, esta ciencia ha incorporado el estudio del turismo como un sistema lo que ha permitido interpretar la multidimensionalidad del fenómeno turístico" (Morera y Miranda, 2015: 21).

El sistema turístico ha sido estudiado desde diferentes perspectivas de la actividad (figura 1); Gunn (2002), visualiza el sistema turístico desde dos variables principales: la oferta y la demanda, planteando que existe una relación invariable entre ellas debido a que, para satisfacer los gustos de la demanda, los gobiernos, regiones o comunidades están dispuestas 
a proveer una variedad de desarrollos y servicios por medio de la oferta en el destino turístico y conformada por: atractivos, transporte, servicios, información y promoción.

\section{Figura 1. Sistema Turístico}

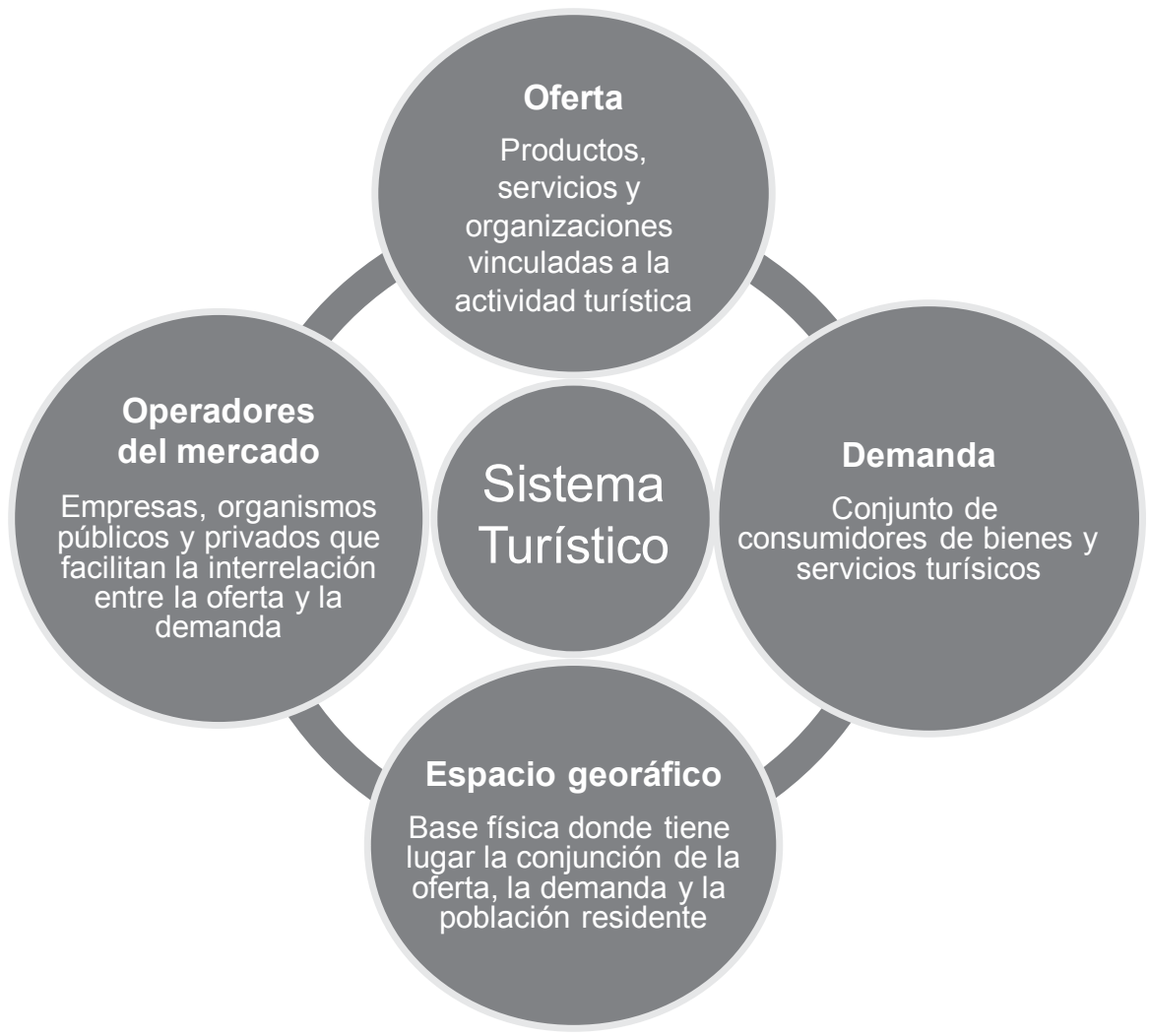

Fuente: Elaboración propia a partir de Sancho, 1998.

Además, menciona en forma clara los factores externos que influyen directamente en el funcionamiento del sistema turístico: recursos naturales, recursos culturales, organizaciones, financiamiento, trabajo, iniciativas empresariales, comunidad, competencia y políticas gubernamentales. Leiper (1990) lo estudia a partir de tres elementos: la región generadora del viaje, la región de tránsito y el destino; por su parte Sancho (1998) lo enmarca a partir de cuatro componentes de la actividad turística: la demanda, la oferta, el espacio geográfico y los agentes del mercado. 


\section{Resultados}

\section{Planificación territorial de la actividad turística}

Desde la planificación del territorio, el turismo en Costa Rica puede estudiarse desde tres niveles: nacional, municipal y sectorial; sin dejar de lado los actores privados que tienen un papel fundamental en el desarrollo de la actividad. Actualmente la planificación se realiza a través del Plan Nacional de Desarrollo Urbano entendido de acuerdo con la Ley de Planificación Urbana, como el conjunto de mapas, gráficos y documentos, que describen la política general de distribución demográfica y usos de la tierra, fomento de la producción, prioridades de desarrollo físico, urbano-regional y coordinación de las inversiones públicas de interés nacional (Miranda, 2005).

Dicho Plan debe incluir estudios técnicos sobre el factor de población, con proyecciones de crecimiento a nivel nacional, regional y urbano, sobre el uso de la tierra con planes sobre la extensión y formas de aprovechamiento de las porciones requeridas para el desarrollo urbano, desarrollo industrial, vivienda y renovación urbana, servicios públicos y ubicación en general de los proyectos sobre transportes, entre otros (Barrantes y Miranda, 2002).

Para los gobiernos locales, el instrumento de planificación urbana es el Plan Regulador, descrito en la Ley 4240, como el instrumento de planificación local de carácter técnico administrativo, producto de un proceso continuo de planeación que incorpora los objetivos nacionales y regionales del desarrollo urbano, y que los concretiza en políticas, instrumentos y acciones que, a nivel municipal, tiendan a reforzar los objetivos anteriores y a lograr un desarrollo equilibrado de sus centros de población en el corto, mediano y largo plazo (Miranda, 2005).

El Parque Nacional Rincón de la Vieja se localiza en los cantones de Upala y Liberia siendo en este último donde se encuentran los dos sectores de acceso a los visitantes (Pailas y Santa María) por lo que el plan regulador directamente relacionado es el correspondiente al cantón de Liberia. Sin embargo este plan regulador fue aprobado de forma parcial (solo para el área urbana de la ciudad de Liberia), dejando las áreas rurales del cantón (entre ellas las comunidades cercanas al área de estudio) sin objetivos ni instrumentos específicos de planificación territorial, afectando directamente el desarrollo de actividades turísticas en dichas zonas. 
A nivel sectorial, el Instituto Costarricense de Turismo desde el año 2001 establece dos dimensiones de planeamiento: la planificación física del espacio turístico, que se relaciona con el manejo del espacio turístico y directamente con el ordenamiento territorial que es básico para promover un desarrollo turístico equilibrado en lo ambiental, lo social y lo económico y la planificación estratégica que se relaciona con el manejo de las variables de competitividad, particularmente con diferenciación, producto y comercialización; a partir de estas dimensiones se delimitan unidades de planeamiento que representan espacios geográficos con características particulares en las que se posibilita en forma macro, un desarrollo turístico determinado por factores ambientales, sociales, culturales, económicos y políticos (ICT, 2002).

El área de estudio se encuentra dentro de la Unidad de Planeamiento Guanacaste Norte (Mapa 2) cuyo desarrollo se propone a partir de dos centros de distribución que son Liberia y Santa Cruz, junto con dos centros turísticos secundarios: La Cruz - El Coco y Tamarindo. Los principales productos son el de sol y playa, aventura y deportes; existe una demanda creciente de productos de naturaleza y aventura tales como el deslizamiento entre la copa de los árboles y la observación activa de flora y fauna terrestre y marina. Entre los principales atractivos se encuentran los parques nacionales de Santa Rosa, Rincón de la Vieja, el Bosque Tropical Seco, playa Naranjo, el Coco, Tamarindo y Panamá. (ICT, 2007). 
Mapa 2. Unidad de Planeamiento Turístico Guanacaste

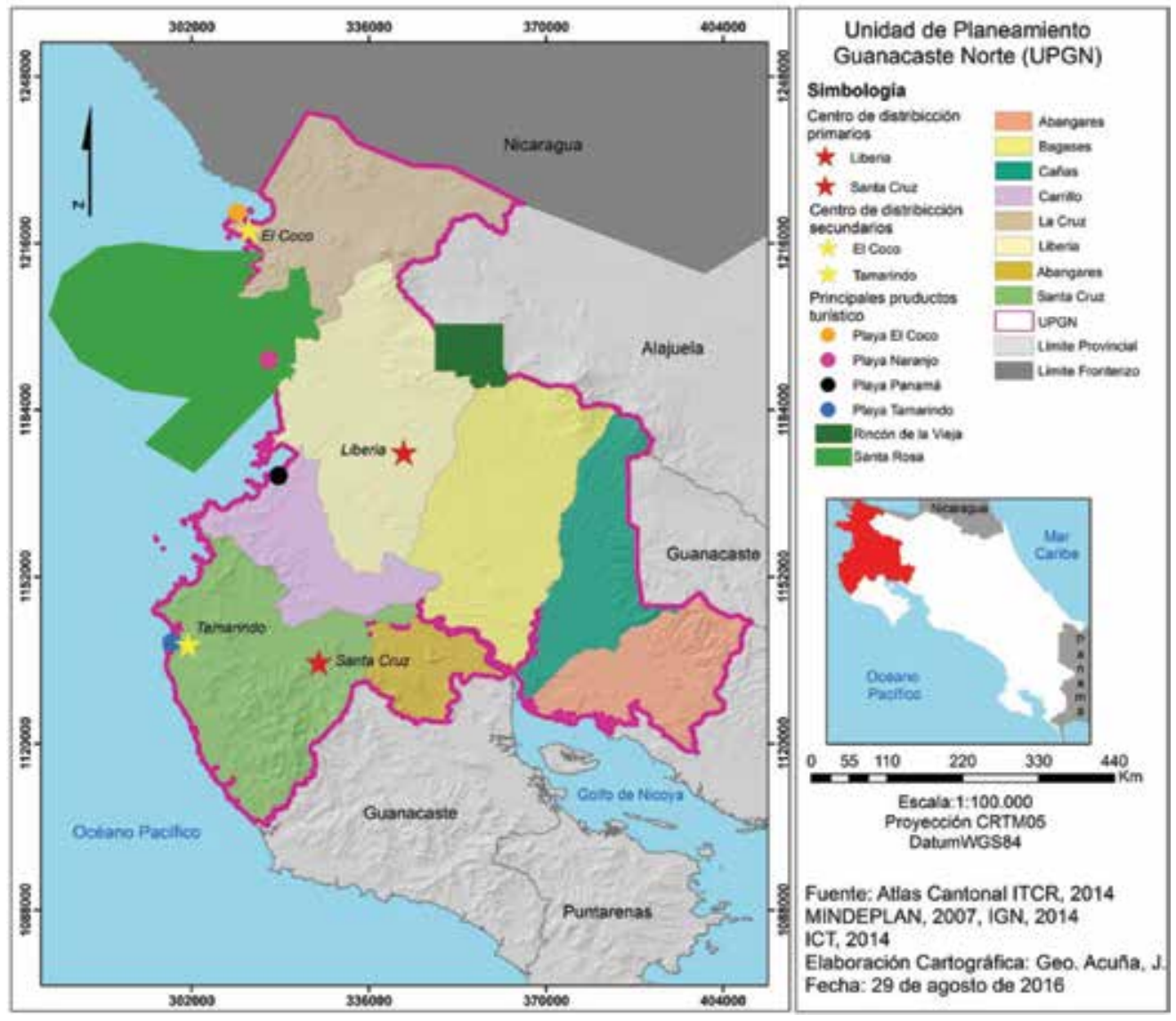

Por su parte el Sistema Nacional de Áreas de Conservación, establecido en el año 1998 mediante la ley de Biodiversidad, agrupa todas las áreas protegidas en once áreas de conservación con el fin de establecer los mecanismos para la administración, manejo y protección de las áreas silvestres protegidas del país, el PNRV forma parte del Área de Conservación Guanacaste declarada por la UNESCO como sitio de patrimonio natural, en el año 1999; cuenta con un único Plan de Manejo elaborado en el año 2014, cuyo objetivo principal es "dirigir el manejo del Área de Conservación Guanacaste en función de un conjunto de elementos focales de manejo, sus principales amenazas y las estrategias adecuadas, dirigidas a mejorar o mantener la integridad de esos elementos" (SINAC, 2014). A partir del mismo el Área de Conservación 
Guanacaste (ACG), ha realizado su gestión desde tres ejes: programas técnicos como ecoturismo, investigación y educación biológica; gerencia de áreas silvestres protegidas encargada de los sectores establecidos para visitación, educación e investigación y gerencia de manejo de recursos naturales.

En el caso del programa de turismo sostenible del ACG, las actividades se centran en seis áreas de acción: atención a visitantes; información y divulgación; facilitación y coordinación de actividades histórico-culturales; planificación y ordenamiento; estaciones biológicas y ordenación procesos que involucren las comunidades e instituciones locales (SINAC, 2015). Bajo este marco, junto con el Programa de Turismo Sostenible financiado por el Banco Interamericano de Desarrollo (BID) para la consolidación del turismo en las áreas silvestres protegidas del país por medio de la gestión sostenible, el desarrollo socioeconómico local y la conservación de los recursos naturales (BID, 2004) y la Estrategia de Turismo Sostenible del SINAC, se elabora el Plan de Turismo Sostenible del PNRV con la finalidad de "incrementar la afluencia al PNRV y proporción de visitantes de la Unidad de Planeación Turística Guanacaste Norte que visitan el parque y su entorno de forma que, sin poner en riesgo la conservación de los recursos, se contribuya al fortalecimiento del SINAC y al desarrollo socioeconómico de las comunidades aledañas y el sector turístico privado" (SINAC, 2014:47), mediante los siguientes programas: fortalecimiento de capacidades en gestión turística, desarrollo de productos turísticos, promoción e inteligencia de mercado y de monitoreo del impacto del turismo.

Como elemento fundamental que completa la gestión realizada por los agentes públicos se encuentran los agentes privados, que tienen relación directa e indirecta con el PNRV, entre ellos los tour operadores, encargados de la organización y traslado de los visitantes. Al ser el área protegida más visitada del ACG se encuentran tour operadores a nivel nacional como Swiss Travel y TAM, además de los locales como Los Tres Monos que ofrecen paquetes, no sólo para la visita del área protegida sino para realizar actividades como cañoning, tirolesa y cabalgatas en sitios aledaños a la misma. Además, como soporte a la organización de estos agentes se encuentran las cámaras de turismo de la zona, siendo las más importantes la Cámara de Turismo de Guanacaste (CATURGUA) y la Cámara Liberiana de Turismo (CALITUR), las cuales poseen diferentes programas y proyectos relacionados con la conservación de los recursos naturales de la zona. 
Cuadro 1. Oferta turística del PNRV

\begin{tabular}{|c|c|c|}
\hline Componente & \multicolumn{2}{|c|}{ Descripción } \\
\hline Atractivos & $\begin{array}{l}\text { Sector Pailas: } \\
\text { - Cráter Rincón de la Vieja }(1,806 \\
\text { msnm) } \\
\text { - Cráter Von Seebach }(1,895 \mathrm{msnm}) \\
\text { - Belleza escénica } \\
\text { - Manifestaciones geotermales } \\
\text { - Gran diversidad de vegetación } \\
\text { - Gran variedad de fauna } \\
\text { - Rios y quebradas } \\
\text { - Cataratas }\end{array}$ & $\begin{array}{l}\text { Sector Santa Maria: } \\
\text { - La Casona } \\
\text { - Pozas de aguas termales (sendero } \\
\text { - Azufrales) } \\
\text { - Aguas burbujeantes (no termales) } \\
\text { - Rios y quebradas } \\
\text { - Una catarata } \\
\text { - Gran diversidad de vegetación } \\
\text { - Gran variedad de fauna } \\
\text { - Bosques } \\
\text { - Bosque de robles (árboles gigantes) }\end{array}$ \\
\hline Actividades & \multicolumn{2}{|c|}{$\begin{array}{l}\text { - Caminatas de moderada intensidad } \\
\text { - Caminatas de alta intensidad y mayor dificultad } \\
\text { - Recorridos autoguiados } \\
\text { - Observación de fenómenos volcánicos } \\
\text { - Observación de flora } \\
\text { - Observación de fauna } \\
\text { - Observación de aves } \\
\text { - Observación de paisajes } \\
\text { - Toma de fotografias o videos aficionados }\end{array}$} \\
\hline Facilidades & $\begin{array}{l}\text { Sector Pallas: } \\
\text { - Sendero circular Las Pailas } \\
\text { - Senderos Catarata La Cangreja y } \\
\text { Catarata Escondida. } \\
\text { - Sendero Copelares-Crater } \\
\text { - Sendero Los Gigantes del Rincón } \\
\text { (Pailas-Santa Maria, } 8 \text { km) }\end{array}$ & $\begin{array}{l}\text { Sector Santa Maria: } \\
\text { - Area de recepción en La Casona } \\
\text { - Sendero y Mirador Santa Maria } \\
\text { - Sendero El Colibri (corto, circular, mide } \\
800 \mathrm{~m}) \\
\text { - Sendero Azufrales }(3,5 \mathrm{~km}) \\
\text { - Sendero Los Gigantes del Rincón (Pailas. } \\
\text { Santa Maria, } 8 \mathrm{~km}) \\
\text { - Sendero Pailas de agua fria } \\
\text { - Sendero Catarata del Bosque Encantado }\end{array}$ \\
\hline Servicios de apoyo & $\begin{array}{l}\text { Sector Pailas: } \\
\text { - Caseta de control de ingresos de } \\
\text { visitantes } \\
\text { - Estacionamiento } \\
\text { - Agua potable } \\
\text { - Servicios sanitarios } \\
\text { - Rotulación }\end{array}$ & $\begin{array}{l}\text { Sector Santa Maria: } \\
\text { - Recepción } \\
\text { - Estacionamiento sin seflalizar } \\
\text { - Agua potable } \\
\text { - Servicios sanitarios } \\
\text { - Area de acampar }\end{array}$ \\
\hline
\end{tabular}

Fuente: SINAC, 2014

\section{Recursos e infraestructura del Parque Nacional Rincón de la Vieja}

Establecido en el año 1974, mediante la ley 5398, este parque cuenta actualmente con un área de 14160 hectáreas y dos sectores abiertos al público (Pailas y Santa María) por medio de los cuales los visitantes tienen acceso a los diferentes recursos y atractivos del área protegida (figura 1); destacan su flora, fauna y las diversas estructuras volcánicas y geotermales e hidrológicas ubicadas en el área protegida. Dentro de las actividades que pueden realizarse, actualmente, por parte de los visitantes se cuentan 
caminatas, acampada, observación de flora, fauna y estructuras geomorfológicas para las cuales se prestan facilidades como zona de recepción, senderos, agua potable, estacionamiento, servicios sanitarios, rotulación entre otros (SINAC, 2014).

\section{Oferta turística en torno al Parque Nacional Rincón de la Vieja}

En el contexto inmediato del área protegida se ha desarrollado una oferta de alojamientos de mayor o menor nivel, en torno a una serie de atractivos, que incluyen las aguas termales y la naturaleza en general. Según el SINAC (2014) esta oferta se organiza en torno a actividades como el de spa, salud y bienestar, así como aventura en el volcán Rincón de la Vieja. También indica el SINAC, que esta oferta también repercute positivamente con empleo para personas de la zona. Para el cantón de Liberia El ICT, registra 41 servicios de hospedaje (de 3 a 300 habitaciones) clasificados en albergues, hosteles, hoteles, cabinas, posadas rurales y hoteles hacienda, en los que también se ofrece el servicio de restaurante y tours guiados por el PNVRV, los que además poseen algún tipo de certificación turística.

Un 34\% de los hoteles de la zona, cuenta con la Declaratoria Turística, que consiste en declaración de una empresa o actividad de Interés Turístico, luego de cumplir con los requisitos técnicos, económicos y legales señalados en el Reglamento del ICT. Y un $12 \%$ cuenta con el Certificado de Sostenibilidad Turística (CST), que se otorga a las empresas turística, realizan buenas prácticas de sostenibilidad turística, integrando los diferentes ámbitos de la sostenibilidad (entorno Físico Biológico, Socioeconómico, Cliente Externo y Planta de Servicio) De acuerdo al puntaje de la evaluación se otorga de 1 a 5 niveles de sostenibilidad, que se asocian a una estructura gradual de incentivos que las empresas podrán disfrutar; a mayor nivel de sostenibilidad mayores beneficios en promoción, capacitación, respaldo para participación en ferias y otros eventos, información entre otros.

Los hoteles que cuentan con el CST, se ubican en las comunidades cercanas al PNVRV, porque ofertan al mismo como su principal recursos turísticos, además implementa las buenas prácticas basada en el uso y conservación de este como su entorno físico biológico, El siguiente mapa, muestra la distribución de estos hoteles, en los alrededores del PNVRV.

Revista Geográfica de América Central Nº Especial ISSN 1011-484X, I Semestre 2017, pp. 185-207 
En cuanto a servicios básicos, únicamente existen 10 negocios locales que además brindar servicios a la población local, lo hacen al turista, como sodas (restaurantes pequeños) pulperías (tiendas) bazares o tiendas de artesanías o regalos.

Mapa 3. Distribución de hoteles en el cantón de Liberia, Guanacaste

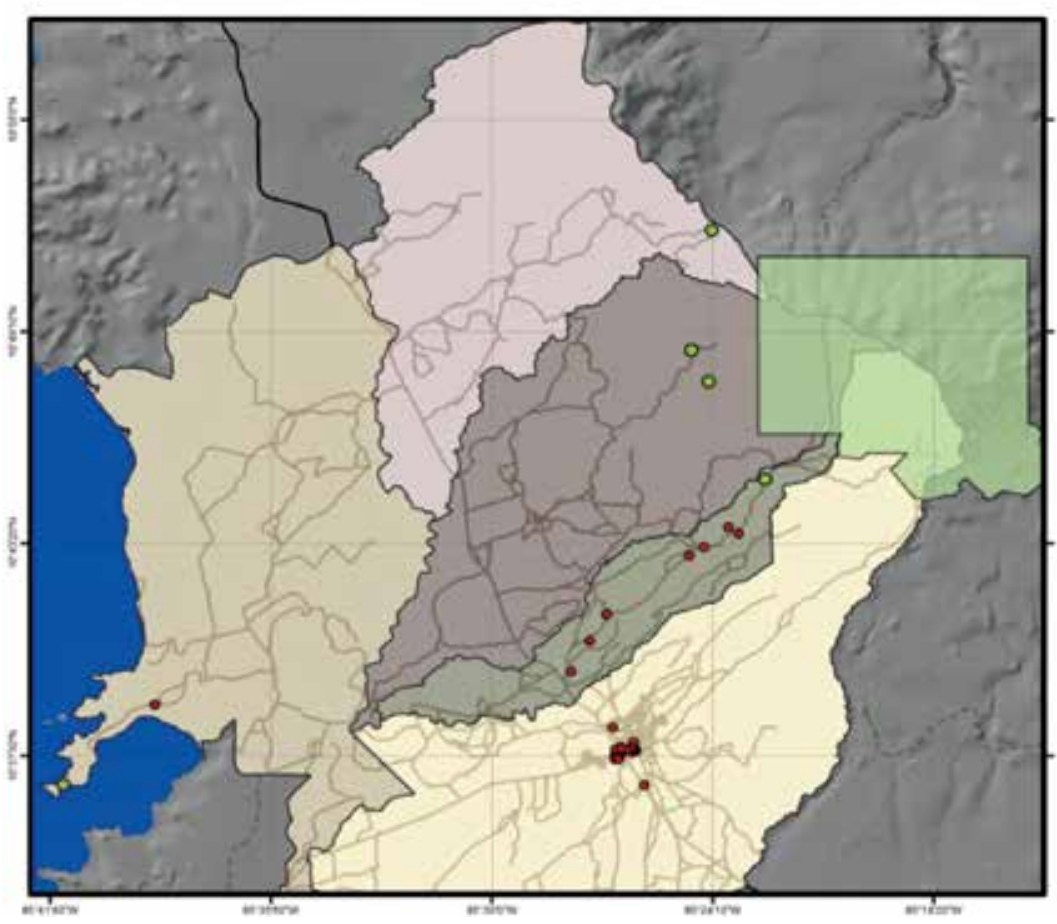

Simbología

- Hoteles

Hoteles con CST

- Red de caminos

PN Rincón de la Vieja

Distritos

$\square$ Canas Duices

$\square$ Curubande

Liberia

$\square$ Mayorga

Nacascolo

Limite distrital

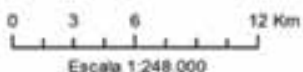

Eacala 1248000

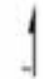

$\begin{array}{lll}\text { Proyeceion GSC wos } & 84 \\ \text { Datum } & \text { Wos } & 84\end{array}$ Fuente Datos de Moleles, ICT, 2013 Coberturas Red de eaminos. Asas ifCR Cober 2000

Cobertura Areas Pnotegidas de Costa Rica, Sinic 2008 Proyecto: Turiamo y Teentorio de breas procegdas de Menico y Amenica Centra Elaboración y diseha Ceda Karta Mora Setiembere 2013 Aparicio

\section{Perfil del turista}

Los consumidores de los bienes y servicios turísticos del PNVRV son, en su mayoría extranjeros; de acuerdo con las estadísticas del ICT, sobre las visitas a las áreas protegidas del Sistema Nacional de Áreas de Conservación (SINAC), en el año 2013, el parque recibió un total de 55.086 personas, de las que $80 \%$ eran extranjeros. El SINAC (2014), con base en diferentes fuentes, hace una caracterización de los turistas internacionales y los turistas nacionales que ingresan al PNVR, el siguiente cuadro las muestra 
Pablo Miranda-Álvarez, Meylin Alvarado-Sánchez. Territorial relations of the tourist activity around Rincón de la Vieja National Park, Costa Rica

Cuadro 2. Características de turistas internacionales y nacionales del PNVRV

\begin{tabular}{|l|ll|}
\hline \multicolumn{1}{|c|}{ Tipo de turista } & \multicolumn{1}{c|}{ Caracterización } \\
\hline & II & Son cerca del 80\% \\
& II & Llegan bien informados acerca de la oferta del PNRV \\
& II & Muchos llegan por recomendación \\
& II & Motivaciones principales de naturaleza y aventura \\
II & Generalmente en buena condición fisica \\
& II & Muchos llegan por turoperadoras (TAM, Swiss Travel, otras) y hoteles (Rincón de la \\
& Vieja Lodge, Hacienda Guachipelín)
\end{tabular}

Fuente: SINAC, 2014.

Los extranjeros que visitan el PNVRV ingresan al país por el aeropuerto Internacional Daniel Oduber, ubicado en la Liberia, Guanacaste. Este aeropuerto recibe quince vuelos diarios ${ }_{2}$ Según el ICT, en 2012, ingresaron 331.116 turistas al aeropuerto de Liberia y para el primer semestre del 2013 llegaron cerca de 143.000. Estos turistas proceden principalmente de Norte América, de Europa y en menor cantidad del resto del continente americano. El siguiente cuadro, muestra la cantidad de visitantes de acuerdo con la región de origen, que ingresaron por dicho aeropuerto en 2012.

200 Revista Geográfica de América Central No Especial ISSN 1011-484X, I Semestre 2017, pp. 185-207 
Cuadro 3. Ingreso de Turistas por el Aeropuerto Internacional Daniel Oduber para el año 2012.

\begin{tabular}{|l|c|}
\hline \multicolumn{1}{|c|}{ Región } & Aeropuerto Daniel Oduber \\
\hline Caribe & 453 \\
\hline América Central & 1539 \\
\hline América del Sur & 2446 \\
\hline Otras zonas & 2900 \\
\hline Europa & 12432 \\
\hline Norte América & 311346 \\
\hline Total & $\mathbf{3 3 1} \mathbf{1 1 6}$ \\
\hline
\end{tabular}

Fuente: Elaboración propia a partir de información proporcionada por la oficina de Migración y Extranjería de Costa Rica, en el 2013.

Los datos anteriores se complementan con la información proporcionada por el Aeropuerto Daniel Oduber, que se presenta en el siguiente mapa sobre la cantidad de vuelos internacionales que llegan a este aeropuerto y el país de origen. En éste se nota que la mayoría de los vuelos proceden de Estados Unidos y Canadá, y en menor cantidad de El Salvador y Panamá.

El PNRV, es una de las 10 ASP del SINAC con mayor visitación de turistas no residentes; se posiciona en el lugar número 5. Esto es por la relativa cercanía con la ciudad de Liberia y su Aeropuerto Internacional "Daniel Oduber Quirós" y con las playas de Guanacaste Norte, el principal destino turístico de Costa Rica. 
Pablo Miranda-Álvarez, Meylin Alvarado-Sánchez. Territorial relations of the tourist activity around Rincón de la Vieja National Park, Costa Rica

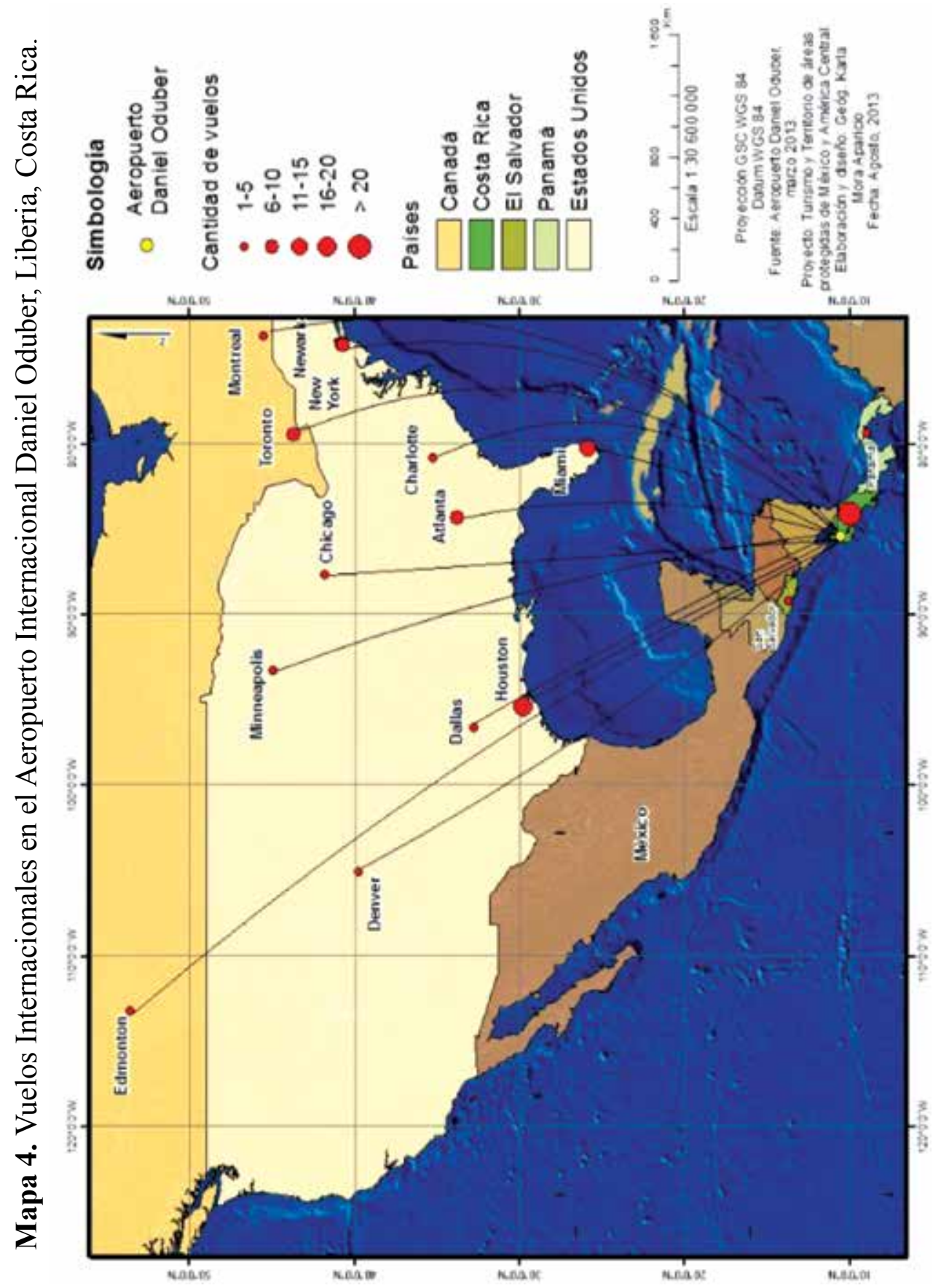

202 Revista Geográfica de América Central No Especial ISSN 1011-484X, I Semestre 2017, pp. 185-207 


\section{Comunidades locales}

En la Propuesta estratégica del Plan de Turismo Sostenible y sus programas de fortalecimiento de capacidades y gestión del turismo, se manifiesta la la En cuanto a la relación con las comunidades por parte de las ASP, Las políticas públicas apuntan a la integración de las comunidades locales en la gestión del turismo, en torno a las ASP, mediante las. En el PNRV, la comunidad que recibirá mayor beneficio con estas propuestas, sería Curubandé de Liberia, por ser el área de acceso al parque y sus principales atractivos.

Se han creado diferentes instrumentos que buscan esto. Por ejemplo la Ley de Biodiversidad, Ley número 7788, plantea la figura de los Consejos locales como organismo para coordinar la visión local y la de las ASP pero en la práctica esta figura está siendo poco empleada lo que en algunos Parques ha provocado el recelo de las comunidades Aledañas y se han creado roses entre estos, generando conflictos como los que se han presentado en el Parque Nacional santa Rosa, con los cazadores ilegales y los incendios provocados al área protegida.

En cuanto a la inclusión de las comunidades aledañas al PNRV, en su Plan de Turismo Sostenible mediante el programa de fortalecimiento de capacidades en gestión turística se busca una mayor integración con la comunidad local. Se trata, por tanto, de fortalecer el conocimiento de la actividad y del mercado turístico y mejorar las competencias de los recursos humanos de las ASP del SINAC en materia de gestión y operación de la actividad turística para que se dé un manejo y una práctica eficaz del turismo.

En la zona de influencia del ASP, el PNRV propiamente se localiza en los cantones de Liberia (distritos de Mayorga, Cañas Dulces y Curubandé) y Upala (distritos de Dos Ríos y Aguas Claras) comunidades que se podrían beneficiar directamente de la existencia del Parque, a través de la oferta de servicios de alimentación, hospedaje, venta de recuerdos y servicios de guiado al turista.

Mediante las estrategias de conservación, aprovechamiento o administración de los recursos naturales y culturales, se generan oportunidades de empleo local, que permiten la permanencia de los habitantes en sus comunidades. 


\section{Conclusiones}

Dentro de los factores que inciden en el alto potencial turístico del Parque Nacional Volcán Rincón de la Vieja, están, su ubicación dentro de la Unidad de Planeamiento Turístico Guanacaste Norte, la unidad de planeamiento, con mayor desarrollo de Costa Rica, la cercanía a un aeropuerto internacional, la proximidad a la carretera Interamericana Norte y a la ciudad de Liberia, donde se concentran los diferentes servicios turísticos.

Dentro de la Unidad de Planeamiento Turístico Guanacaste Norte, también se ubican los principales destinos de sol y playa del país, por lo que este parque tiene la función de complementar el turismo masivo con un tipo de turismo diferente como el ecoturismo, o el turismo de volcán y naturaleza, que por sus característica, le permite a los empresarios locales, la implementación de la sostenibilidad turística, aportando al desarrollo local de las comunidades de la siguiente manera:

- Mediante estrategias de conservación, aprovechamiento o administración de los recursos naturales y culturales, se generan oportunidades de empleo local, que permiten la permanencia de los habitantes en sus comunidades.

- Incentivan la participación de las comunidades en la actividad turística, mediante el encadenamiento de servicios y venta de productos locales, lo que les brinda un nuevo soporte para la competitividad del sector empresarial,

- La implementación de Buenas Prácticas de Sostenibilidad Turísticas pueden contribuir en un futuro proceso de ordenamiento territorial municipal, porque el CST, exige que las empresas tengan procesos de concienciación de las comunidades sobre la importancia de la conservación, además de propiciar la participación ciudadana en el proceso de ordenamiento territorial.

Las políticas públicas apuntan a la integración de las comunidades locales en la gestión del turismo, en torno a las ASP, mediante las Propuesta estratégica del Plan de Turismo Sostenible y sus programas de fortalecimiento de capacidades y gestión del turismo. En el PNRV, la comunidad que recibirá mayor beneficio con estas propuestas, sería Curubandé de Liberia, por ser la comunidad más cercana y el único acceso al parque y sus atractivos.

Revista Geográfica de América Central Nº Especial ISSN 1011-484X, I Semestre 2017, pp. 185-207 


\section{Referencias}

Asamblea Legislativa de la República de Costa Rica. (2007). Ley para el fomento del turismo rural comunitario. San José: Departamento de Servicios Parlamentarios.

Banco Interamericano de Desarrollo. (2004). Programa de turismo en áreas silvestres protegidas. Recuperado de http://idbdocs.iadb.org/ wsdocs/getdocument.aspx?docnum $=880775$.

Barrantes, L. \& Miranda, P. (2002). Diagnóstico y zonificación de las variables físico - geográficas como base para la planificación del ordenamiento territorial en los distritos de Heredia, Mercedes, San Francisco y Ulloa, cantón central de Heredia. Trabajo de graduación licenciatura. Escuela de Ciencias Geográficas, Universidad Nacional de Costa Rica. (pp. 350)

Denyer., P. \& Kussmaul, S. (2000). Geología de Costa Rica. Cartago, Costa Rica: Editorial Tecnológica de Costa Rica.

Dudley, N. (ed). (2008). Directrices para la aplicación de las categorías de gestión de áreas protegidas. Glad, Suiza: UICN.

Goeldner, C. \& Ritchie, J. (2011). Turismo: planeación, administración y perspectivas. México: Limusa Wiley.

Gunn, C. \& Var, T. (2002). Tourism planning: basics concepts and cases. USA: Routledge.

Instituto Costarricense de Turismo. (2015). Anuario estadístico del turismo. San José, Costa Rica: ICT. Recuperado de http://www.visitcostarica.com/ict/pdf/estadisticas/Anuario_de_Turismo_2014.pdf.

Instituto Costarricense de Turismo. (2007). Unidad de planeamiento Guanacaste Norte: plan de uso del suelo y desarrollo turístico. Recuperado de http://www.ict.go.cr/es/documentos-institucionales/plan-nacional-y-planes-generales/planes-generales-por-unidad-de-planeamiento/guanacaste-norte/132-plan-4/file.html.

Instituto Costarricense de Turismo. (2002). Plan General de Desarrollo Turístico Sostenible 2002-2012. San José, Costa Rica: ICT.

Instituto de Fomento y Asesoría Municipal. (1989). Atlas Cantonal. San José, Costa Rica: IFAM.

Instituto Geográfico Nacional. (1978). Mapa geomorfológico de Costa Rica, escala 1:200.000, hoja Liberia. San José, Costa Rica: IGN 
Instituto Nacional de Estadística y Censos (2012). X censo nacional de población y VI de vivienda 2011: características sociales y demográficas. San José, Costa Rica: INEC.

Instituto Tecnológico de Costa Rica. (2000). Atlas digital 2000. Cartago, Costa Rica: ITCR.

Leiper, N. (1990). Tourism systems. An interdisciplinary perspective. Occasional Papers 1990 (2). New Zealand: Massey University.

Lozato-Giordat, J.C. (1990). Del espacio contemplado al espacio consumido. Barcelona, España: Mansson, SA.

Miranda, P. (2005). Propuesta de plan estratégico de desarrollo turístico para el cantón de San Rafael, Heredia. Trabajo de graduación maestría. Escuela de Ciencias Geográficas, Universidad Nacional, Costa Rica. (pp. 155)

Monterrubio, J. (2011). Turismo y cambio sociocultural: una perspectiva conceptual. México: Plaza y Valdés Editores.

Morera, C. \& Miranda, P. (2015). De la geografía del turismo al análisis territorial del turismo: el rastro en Costa Rica. En: Revista Geográfica de América Central, 54, 15-43.

Programa Naciones Unidas para el Desarrollo. (2011). Atlas del desarrollo humano cantonal de Costa Rica 2011. San José, Costa Rica: PNUD-UCR.

Quirós, L., Alfaro, C. y Miranda, P. (2014). El turismo en los volcanes de Costa Rica: el caso del Parque Nacional Volcán Poás. En A. Sánchez \& L. Quirós, (Ed.). Volcanes y ecoturismo en México y América Central (pp.273-302). Costa Rica: EUNA.

Sancho, A., Cabrer, B., García, G. y Pérez, J. (2001). Apuntes de metodología de la investigación en turismo. España: Organización Mundial del Turismo.

Sancho, A. (1998). Introducción al Turismo. España: Organización Mundial del Turismo.

SECTUR. (2007). Elementos para evaluar el impacto económico, social $y$ ambiental del turismo de naturaleza en México. México, Distrito Federal.

SECTUR. (2004). Turismo alternativo, una nueva forma de hacer turismo. México, Distrito Federal. 
Sistema Nacional de Áreas de Conservación (2015). Programa de Ecoturismo. Recuperado de http://www.acguanacaste.ac.cr/biodesarrollo/ programa-de-ecoturismo.

Sistema Nacional de Áreas de Conservación. (2014). Plan de turismo sostenible del Parque Nacional Rincón de la Vieja. Recuperado de http://www.acguanacaste.ac.cr/noticias/noticias-programa-de-ecoturismo/892-plan-de-turismo-sostenible-del-pnrv-a-la-pagina-del-acg.

Valls, J., Bustamante, X., Gúzman, F. y Vila. M. (2004). Gestión de destinos turísticos sostenibles. Barcelona: Gestión 2000.

Vargas, G. (1997). Geografía turística de Costa Rica. San José, Costa Rica: EUNED.

Velasco, M. (2013). Conceptos en evolución: turismo, cultura y turismo cultural. En Pulido, J (Ed). Turismo Cultural (pp.15-43). España: Editorial Síntesis.

Vera, J., López, F., Marchena, M. y Antón, S. (2013). Análisis territorial del turismo y planificación de destinos turísticos. Valencia, España: Tirant Humanidades. 